\title{
SUB-DEGREE ANGLE DETECTION USING MICROMACHINED DOME-SHAPED- DIAPHRAGM RESONATOR WITH WINE-GLASS MODE VIBRATION
}

\author{
A. Vafanejad and E.S. Kim \\ Department of Electrical Engineering, University of Southern California \\ Los Angeles, CA 90089
}

\begin{abstract}
A sensitive, high quality factor (Q), 3-D piezoelectric microelectromechanical systems (MEMS) gyroscope based on wine-glass mode vibration has been designed and fabricated. Experimental results show the functionality of the device as hemispherical rate integrating gyroscope (HRG). The gyroscope utilizes a 1.5-mm-diameter spherical shell made of a LPCVD silicon nitride diaphragm. When the spherical shell is piezoelectrically actuated to vibrate as a four-node wine-glass mode at $126 \mathrm{kHz}$ through a set of four piezoelectric drive elements, an applied angular rotation causes a change of the mode shape, which is piezoelectrically sensed through a set of four piezoelectric sense elements located at $45^{\circ}$ off from the drive elements. A sputter-deposited piezoelectric zinc oxide $(\mathrm{ZnO})$ thin film on the diaphragm is used to actuate and sense vibration at the rim of the fabricated dome. The vibration mode is measured to have a Q of about 10 million, and decays at a time constant of 23 seconds when the piezoelectric actuation is halted. With it, the minimum detectable angle of rotation is measured to be about $0.15^{\circ}$.
\end{abstract}

\section{INTRODUCTION}

Most vibratory gyroscopes measure angular rate in $\% / \mathrm{sec}$, and the net angle measurement is obtained by time integration of the measured angular rate. Such rate gyroscopes that have extensive applications in automotive, aerospace and consumer electronics have several drawbacks. Noise and bias in the measured angular rate, when integrated, will cause a diverging error in the measured angle [1]. On the other hand, rate integrating gyroscopes measure the net angle of rotation directly. In this paper we describe a rate integrating gyroscope based on a hemispherical dome. Macroscale hemispherical resonator gyroscope (HRG) has already been used for space application [2], and one of the most successful vibratory gyroscopes is Northrop Grumman's HRG [3], which uses inertial precession of a standing wave in an axisymmetric and isotropic resonator shell. However, the manufacturing technology for macroscale HRG does not provide a path to device miniaturization. Consequently, the need for wafer-level manufacturing of microscale spherical shells (to exploit the advantages offered by a rate integrating gyroscope) motivates the device presented in this paper. One of the reasons why microscale HRG had not been actively pursued is the difficulty of fabricating the needed 3D structure. But recent innovations in microfabrication of dome structures $[4,5]$ have made microscale HRG become viable.

Our approach to form HRG is to use isotropic etching of silicon in HNA (HF:Nitric:Acetic) to form a spherical etch cavity in silicon for a molding structure, over which low stress silicon nitride film is deposited. When the silicon is etched from the other side of the wafer, the silicon nitride film forms a hemispherical dome or spherical cap with its boundary supported by the same thin silicon nitride. Unlike other approaches in literature, which use a stem to support the dome diaphragm [6], our approach is to support the dome diaphragm with a thin, flexible membrane that provides room for the circular edge of the dome to vibrate in the second circumferential mode without a need to an anchor or stem. The dome-shaped diaphragm is then piezoelectrically actuated to operate in a 4-node wine-glass mode.

\section{FABRICATION AND ASSEMBLY PROCESS}

Isotropic etching of silicon is done by a combination of $49 \%$ hydrofluoric acid, 70\% nitric acid and 99.5\% acetic acid (HNA) with a ratio of $20: 35: 55$ at $50^{\circ} \mathrm{C}$ [7]. This combination has given the best isotropicity, and we have been able to fabricate large dome diaphragms (up to 1.5 millimeter in diameter) with excellent isotropicity. Figure 1 shows the fabricated transducer. On top of the silicon-nitride dome diaphragm, four piezoelectric sensors and four piezoelectric actuators are formed by depositing $\mathrm{ZnO}$ film and electrode layers through shadow masks. The fabrication steps are briefly described in Fig. 2.

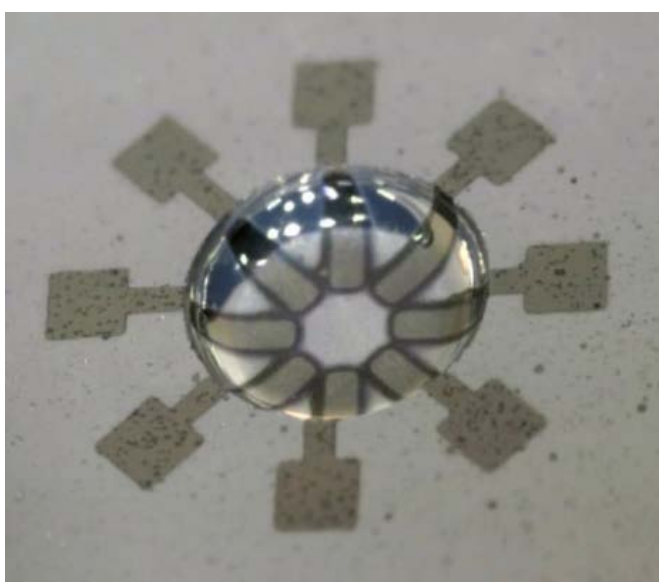

Figure 1: Top-view photo of the fabricated dome-shapeddiaphragm transducer.

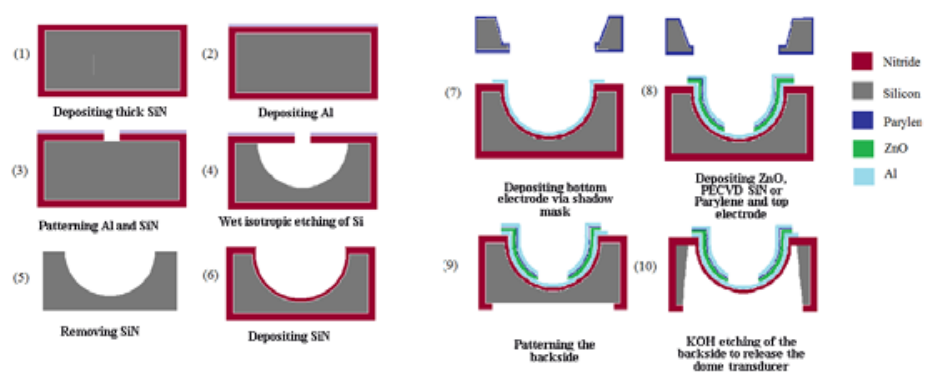

Figure 2: Brief fabrication steps of the dome-shapedtransducer.

\section{SIMULATIONS}

We have simulated the dome diaphragm's mode shapes and resonant frequencies with a finite element analysis, using COMSOL Multiphysics package. Finite element modeling (FEM) of the dome diaphragm resonator having $1.5-\mathrm{mm}$ diameter and an average thickness of $4 \mu \mathrm{m}$ shows a 4-node wineglass mode at a frequency of around $126 \mathrm{kHz}$ (Fig. 3) [4, 7]. 


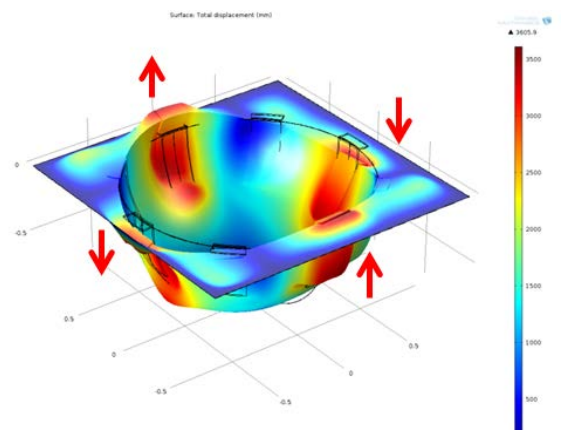

Figure 3: FEM simulation result of the dome-shaped-diaphragm that clearly shows a four-node wine-glass vibration mode.

\section{MEASUREMENTS AND RESULTS}

The fabricated dome-shaped-diaphragm piezoelectric resonator was characterized at a vacuum pressure of $<50 \mathrm{mTorr}$ at room temperature. First, the resonator was mounted on a printed circuit board (PCB), as shown in Fig. 4a and b, which was then placed over a larger PCB that houses the electronic circuits for actuation and sensing.

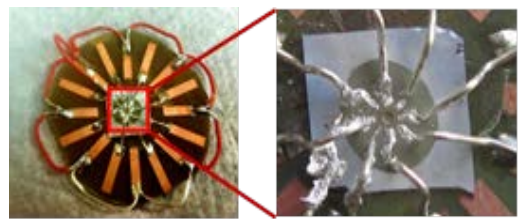

(a)

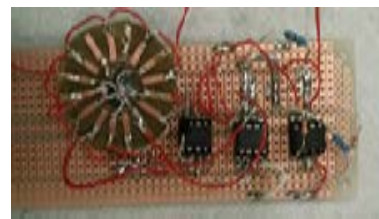

(c)
Figure 4: Photos of the dome diaphragm transducer on a printed circuit board with electrical wires connected to the bonding pads through epoxy: (a) the dome transducer and the whole PCB and (b) a close-up view. (c) Photo of the transducer and electronics for actuation and sensing.

A standing wave in a wine-glass mode is generated by energizing four piezoelectric actuators located on the anti-nodal lines of the wine-glass mode. Two of the four actuators (1 and 5 in Fig. 5) are energized with an identical signal, while the other two ( 3 and 7 in Fig. 5) are actuated by a signal with $180^{\circ}$ phase lag.

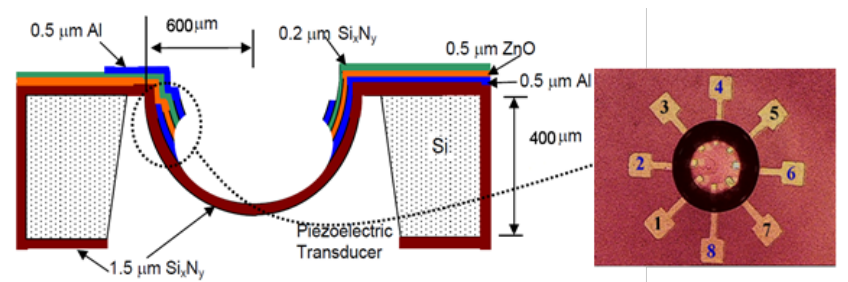

(a)

(b)

Figure 5: (a) Cross-sectional diagram, (b) top view photo of the dome-shaped-transducer that is suspended by a thin flexible membrane and actuated by four piezoelectric transducers and sensed by four other ones, placed on the rim of the dome diaphragm.

The vibration of the dome diaphragm is measured by monitoring the electrical signal output of the piezoelectric sensors embedded on the dome diaphragm rim. A schematic of measurement setup is shown in Fig. 6.

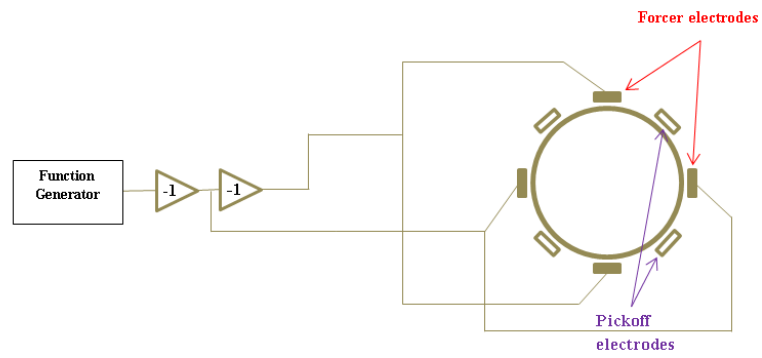

Figure 6: Schematic of the measurement setup.

To confirm the wine-glass mode vibration, we compared the phase difference between the sensor signals and the signals applied to the actuators 1 and 5 in Fig. 5b. Applying electrical signal to a pair of the actuators and picking up the signals from either the Sensor 3 or Sensor 7 (as illustrated in Fig. 8), we observe that the measured signal from the Sensor 3 has $180^{\circ}$ phase difference from the signal applied to the Actuator 1, as can be seen in Fig. 7, as expected.

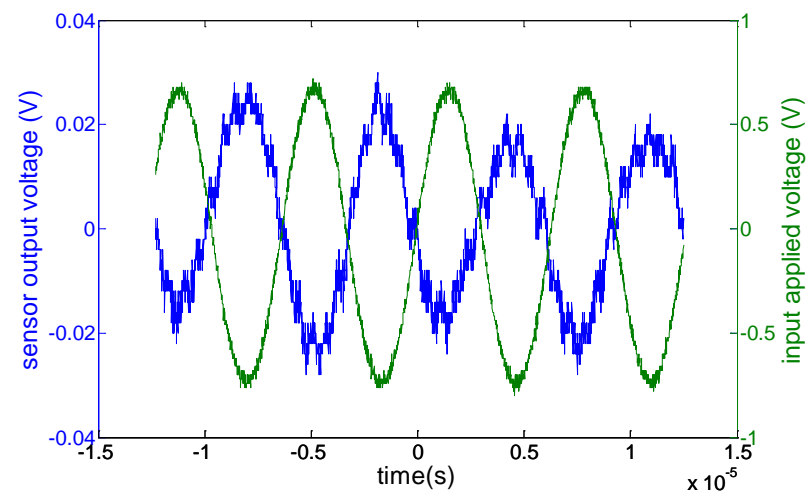

Figure 7: Oscilloscope traces of the applied signal at the Actuator 1 (green curve), measured signal at the Sensor 3 (blue curve). The $180^{\circ}$ phase difference between the two curves shows that the vibration mode is indeed a four-node wine-glass mode.

\section{DECAY TIME CONSTANT MEASUREMENTS}

One important requirement for rate-integrating gyroscopes in aircraft's inertial navigation system is a large decay time constant through a high Q so that the actuating and sensing elements do not have to work simultaneously and also that the measurable range of the rotation angle may be large [2]. We measure the decay time constant of our fabricated dome-shaped-diaphragm resonators by actuating the resonators at their resonance frequencies, stopping the actuation, recording the decaying amplitudes, and fitting the recorded amplitudes with an exponential function, $A_{o} \exp (-t / \tau)$. As can be seen in Fig. 9, it took 23 seconds for the amplitude of the sensed signal to drop by $63 \%$ after the signal to the actuators was turned off. Such a large decay time corresponds to a Q of 8.7x10 ${ }^{6}$.

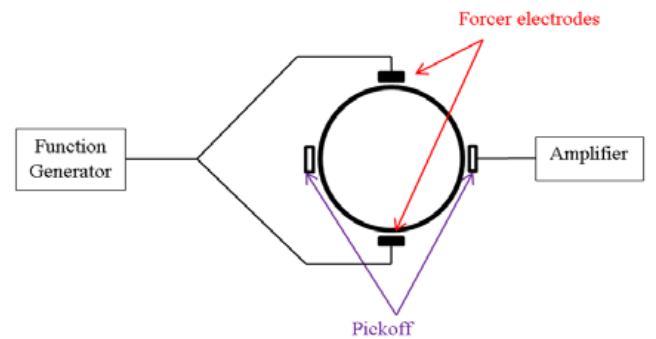

Figure 8: Schematic to illustrate the relative locations for actuation and sensing used to measure the decay time. 


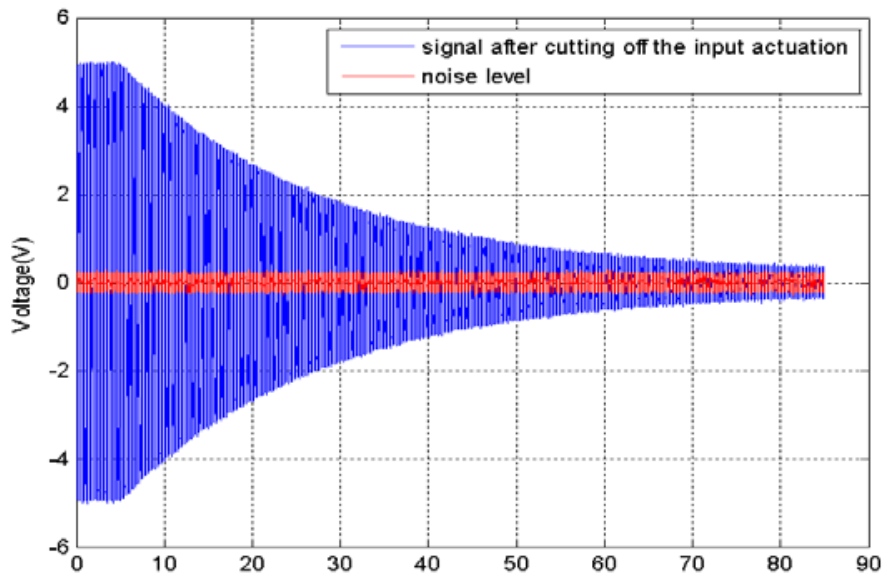

Figure 9: Ring down measurement of the device that shows a large decay time of 23 seconds.

\section{ROTATION MEASUREMENTS}

With our fabricated resonator in a vacuum chamber supported on a rotation table, we measured the resonator's response to rotation through monitoring the output signal at one of the nodal points. As the resonator was rotated at a rate of $1 \%$, the piezoelectrically sensed signal was recorded in a computer after amplification. As can be seen in Fig. 10, the output voltage of nodal point linearly increases as the net angle of rotation is increased (with the angular rate kept constant at about $1 \%$ ). This result shows the functionality of our resonator as a "rate integrating gyroscope".
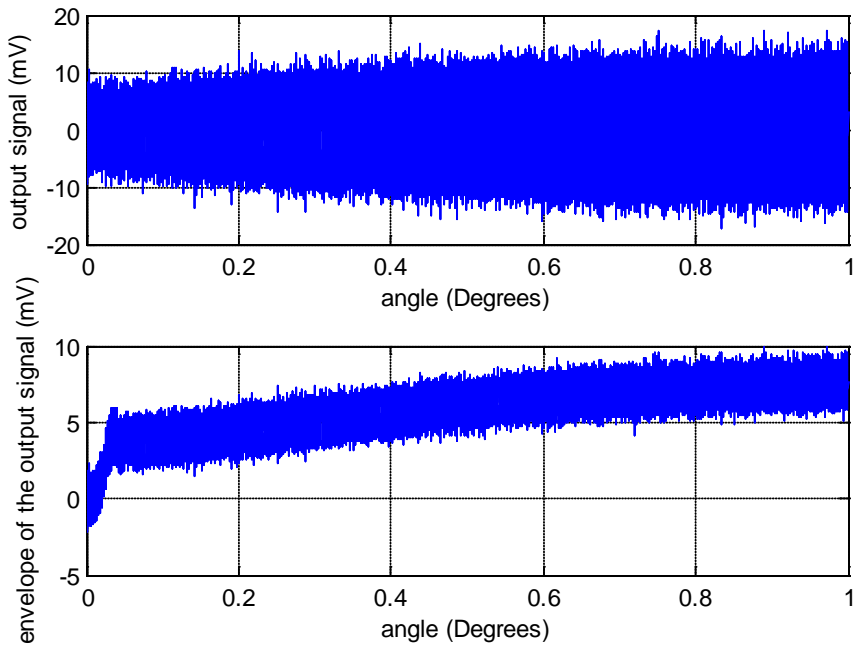

Figure 10: (Top) Measured output signal from a nodal point as a function of the total angle of rotation. (Bottom) Envelope of the measured signal as a function of the total angle of rotation. The large noise level at zero degree is mostly due to electromagnetic interference, and can be reduced substantially through a better packaging technique.

Close-up view on the measured data in Fig. 10 near zero net rotation angle is shown in Fig. 11, from which we see that the minimum detectable angle (or the point where the sensed signal surpasses the noise) is about $0.15^{\circ}$. The sub-degree detectability is highly encouraging for a microfabricated device.

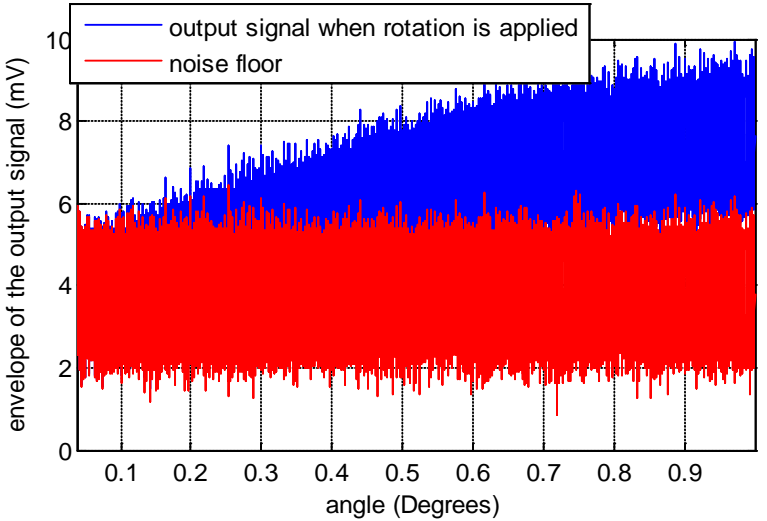

Figure 11: Measured output signal of the transducer when a rotation rate of $1 \%$ is applied. The noise floor limits the minimum detectable angle to be about $0.15^{\circ}$.

\section{RESPONSE TO FORWARD/BACKWARD ROTATIONS}

We have measured how our resonator responds, when we rotate the device in one direction for a time and then reverse the direction of rotation. As depicted in Fig. 12, when the device is rotated for 3 second in one direction and then the direction of rotation is reversed (with a faster rate of change), output voltage of nodal point is also decreasing as the rotation angle is reversed.
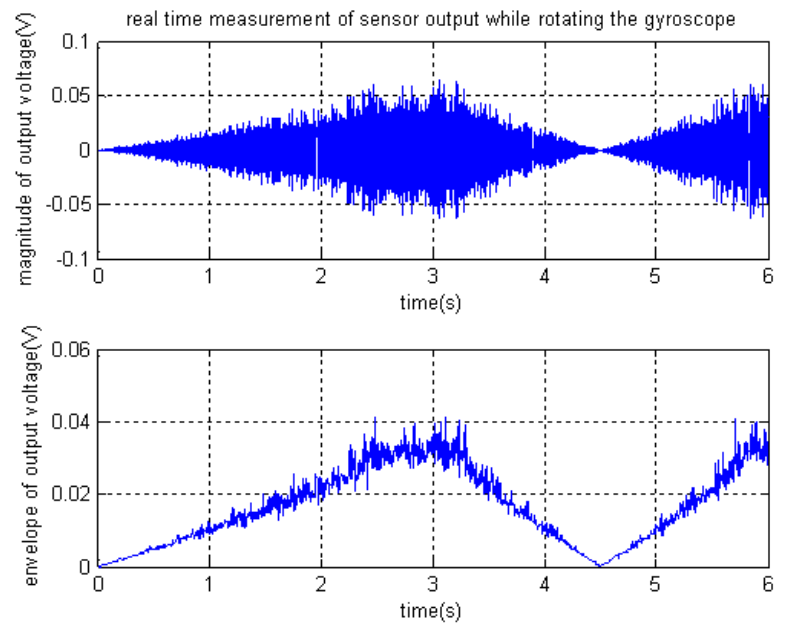

Figure 12: (Top) Measured signal from the sensor when we rotate the device in one direction for a time and then reverse the direction of rotation and (Bottom) the envelope of the measured signal.

By linear fitting of the envelope of the signal and comparing it with the angular rate and absolute angle, we find that the unamplified sensitivity is $4.84 \mathrm{mv} / \%$ s. This sensitivity can be compared with those of published rate-integrating gyroscopes [9, 10]. However, comparison of sensitivities for piezoelectric and capacitive sensing is not straightforward, as the sensitivity of capacitive sensing depends on the polarization voltage (or how the capacitance change is detected), while with piezoelectric sensing unamplified sensitivity can easily be defined due to its capability of producing a voltage difference without any help from a polarization voltage or circuitry.

\section{MINIMUM DETECTABLE RATE OF ROTATION}

We also characterized our device to see what its minimum detectable angular rate in $\%$ is. As can be seen in Fig. 13, our device can detect down to $0.1 \%$. At this point, the minimum detectable angular rate and minimum detectable angle are limited by the relatively large noise level that is mostly due to 
electromagnetic interferences (EMI) that come from the fact that our device has not yet been packaged with an EMI shielding box. It will be relatively straightforward to reduce the EMI by an order of magnitude.
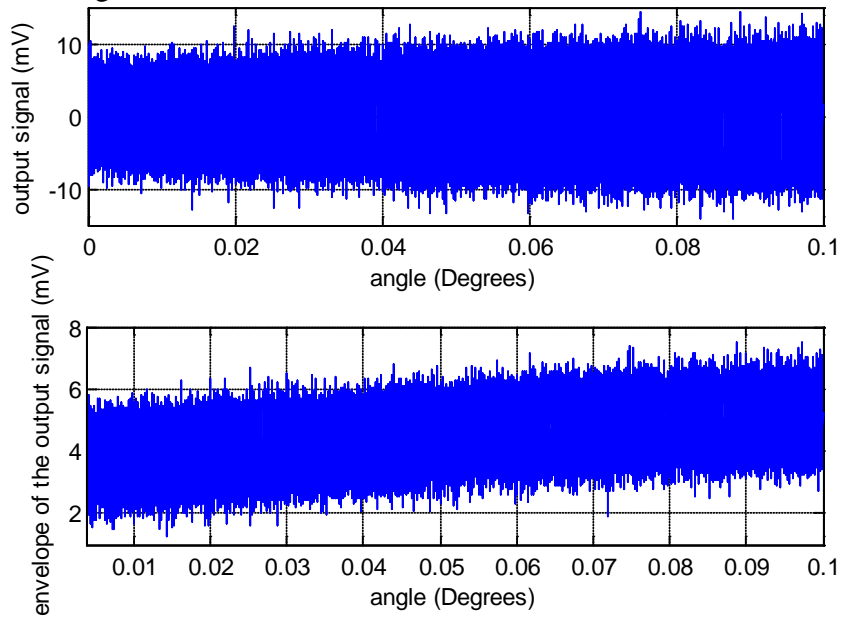

Figure 13: Measured signal (top) and its envelope (bottom), when the device is rotated at an angular rate of $0.1 \%$. At this low rotation rate, the device is capable of picking up signal proportional to the absolute angle of rotation.

\section{SUMMARY}

A wafer-level microfabrication method for a symmetric domeshaped resonator has been developed. The fabricated dome diaphragm is piezoelectrically actuated to vibrate in a four-node wine-glass mode, of which the shape change is sensed piezoelectrically. The micron-sized device shows a very large decay time constant (23 seconds) which is translated to a large Q factor (around 8.7 million), and compares very well with published results (Table 1). With the dome's soft boundary condition, the fabricated device has shown to be functional as a rate integrating gyroscope capable of detecting a sub-degree rotation as well as the direction of the rotation. It also works as an angular rate sensor with minimum detectable angular rate of around $0.1 \%$ s. Currently, the minimum detectable angle and minimum detectable rate of rotation are limited by relatively high electromagnetic interference, which can be reduced substantially through a better packaging technique.

Table 1: Comparison of our device and some reported in literature.

\begin{tabular}{|c|c|c|c|c|c|}
\hline & & $\begin{array}{l}\text { Dome size } \\
\text { in } \\
\text { diameter }\end{array}$ & $\begin{array}{l}\text { Quality } \\
\text { factor }\end{array}$ & $\begin{array}{l}\text { Decay time } \\
\text { constant }\end{array}$ & Sensitivity \\
\hline \multicolumn{2}{|l|}{ Our device } & $\begin{array}{l}1,500 \\
\mu \mathrm{m}\end{array}$ & $8.74 \times 10^{6}$ & $23 \mathrm{~s}$ & $\begin{array}{l}4.8385 \\
\mathrm{mv} / \% / \mathrm{s}\end{array}$ \\
\hline \multirow{3}{*}{$\begin{array}{l}\text { Reported } \\
\text { results in } \\
\text { literature }\end{array}$} & $\begin{array}{l}\text { MEMS } \\
\text { '12 [11] }\end{array}$ & $\begin{array}{l}5,000 \\
\mu \mathrm{m}\end{array}$ & $2.6 \times 10^{5}$ & $8 \mathrm{~s}$ & $\begin{array}{l}27.8 \\
\mathrm{mv} / \% / \mathrm{s}\end{array}$ \\
\hline & $\begin{array}{l}\text { MEMS } \\
\text { '12 [12] }\end{array}$ & $\begin{array}{l}1,200 \\
\mu \mathrm{m}\end{array}$ & 8,000 & $6 \mathrm{~ms}$ & $\begin{array}{l}\text { Not } \\
\text { reported }\end{array}$ \\
\hline & $\begin{array}{l}\text { JMEMS } \\
\text { '12 [13] }\end{array}$ & $\begin{array}{l}1,000 \\
\mu \mathrm{m}\end{array}$ & 470 & $158 \mu \mathrm{s}$ & $\begin{array}{l}2.1 \\
\mathrm{mv} / \% / \mathrm{s}\end{array}$ \\
\hline
\end{tabular}

\section{REFERENCES}

[1] Gallacher, B.J., "Principles of a Micro-Rate Integrating Ring Gyroscope," Aerospace and Electronic Systems, IEEE Transactions on , vol.48, no.1, pp.658,672, Jan. 2012.

[2] Meyer, A. D.; Rozelle, D. M., "Milli-HRG inertial navigation system," Position Location and Navigation Symposium (PLANS), 2012 IEEE/ION , vol., no., pp.24,29, 23-26 April 2012.

[3] Dickinson, J.; Strandt, C.R., "HRG strapdown navigator," Position Location and Navigation Symposium, 1990. Record. The 1990's - A Decade of Excellence in the Navigation Sciences. IEEE PLANS '90., IEEE , vol., no., pp.110,117, 20 23 Mar 1990.

[4] C.H. Han and E.S. Kim, "Fabrication of dome-shaped diaphragm with circular clamped boundary on silicon substrate," Micro Electro Mechanical Systems, 1999. MEMS '99. Twelfth IEEE International Conference on, vol., no., pp.505-510, 17-21 Jan 1999.

[5] Prikhodko, I.P.; Zotov, S.A.; Trusov, A.A.; Shkel, A.M., "Microscale Glass-Blown Three-Dimensional Spherical Shell Resonators," Microelectromechanical Systems, Journal of , vol.20, no.3, pp.691,701, June 2011

[6] Cho, J.; Yan, J.; Gregory, J.A.; Eberhart, H.; Peterson, R.L.; Najafi, K., "High-Q fused silica birdbath and hemispherical 3$\mathrm{D}$ resonators made by blow torch molding," Micro Electro Mechanical Systems (MEMS), 2013 IEEE 26th International Conference on , vol., no., pp.177,180, 20-24 Jan. 2013.

[7] H. Zhang; E.S. Kim, "Dome-shaped diaphragm microtransducers," Micro Electro Mechanical Systems, 1995, MEMS '95, Proceedings. IEEE , vol., no., pp.256, 29 Jan-2 Feb 1995.

[8] C.H. Han and E.S. Kim, "Simulation of Piezoelectric DomeShaped-Diaphragm Acoustic Transducers," Journal of Semiconductor Technology and Science, vol. 5, no. 1, pp. 1723, 2005.

[9] Zotov, S.A.; Trusov, A.A.; Shkel, A.M., "High-Range Angular Rate Sensor Based on Mechanical Frequency Modulation," Microelectromechanical Systems, Journal of , vol.21, no.2, pp.398,405, April 2012.

[10] Gregory, J. A.; Cho, J.; Najafi, K., "Characterization and control of a high-Q MEMS inertial sensor using low-cost hardware," Position Location and Navigation Symposium (PLANS), 2012 IEEE/ION , vol., no., pp.239,247, 23-26 April 2012.

[11] Chan, M.L.; Fonda, P.; Reyes, C.; Xie, J.; Najar, H.; Lin, L.; Yamazaki, K.; Horsley, D.A., "Micromachining 3D hemispherical features in silicon via micro-EDM," Micro Electro Mechanical Systems (MEMS), 2012 IEEE 25th International Conference on , vol., no., pp.289,292, Jan. 29 2012-Feb. 22012.

[12] Sorenson, L.D.; Gao, X.; Ayazi, F., "3-D micromachined hemispherical shell resonators with integrated capacitive transducers," Micro Electro Mechanical Systems (MEMS), 2012 IEEE 25th International Conference on , vol., no., pp.168,171, Jan. 29 2012-Feb. 22012.

[13] Zotov, S.A.; Trusov, A.A.; Shkel, A.M., "Three-Dimensional Spherical Shell Resonator Gyroscope Fabricated Using Wafer-Scale Glassblowing," Microelectromechanical Systems, Journal of , vol.21, no.3, pp.509,510, June 2012.

\section{CONTACT}

*A. Vafanejad, tel: +1-818-515-2793; vafaneja@usc.edu 